\title{
UPM prosthetic hand system design - preliminary results
}

\begin{abstract}
This paper presents the preliminary findings of the active prosthetic hand designed concept for the Universiti Putra Malaysia. The development process is clearly stated in order to successfully designing the hand prosthesis. The classification of the level of amputation and current standard of hand prosthesis are also included in the design process. The design targets for lower-level upper-limb amputees and will be based on electromyography controlled (transhumeral amputation).
\end{abstract}

Keyword: EMG; Non-surgical prosthetic; Prosthetic hand; SEMG 